\title{
Une Approche Discursive de la Classe de langue Étrangère en tant que LieU de Travail
}

\author{
(A Discoursive Approach of Foreign Language \\ Classroom as a Place of Work)
}

Décio RochA

(Universidade do Estado do Rio de Janeiro)

\begin{abstract}
This paper focuses the analysis of speech in foreign language classroom in a particular situation: the teaching of French as a professional language. Our purpose will be to explore enunciative marks that contribute to determine the nature of the work carried out in classroom throughout the collective construction of a text which is built by the actors of the didactic scene, the teacher and the students. In order to achieve our goal, we will interrogate these discourses from a triple point of view: first of all, the opposition between collective work and individual work in classroom; afterwards, the double function of language - language as work and language about work - in such a context; finally, the distance verified between prescribed work and real work in classroom.
\end{abstract}

KEY-WORDs: Classroom speech; Enunciation; Work.

Resumo: O presente artigo centra-se na análise dos discursos da aula de língua estrangeira em uma situação particular: o ensino da língua francesa como língua profissional. Nosso objetivo consistirá em depreender marcas enunciativas por intermédio das quais se inscreve a natureza do trabalbo realizado em sala de aula, tendo em vista a construção coletiva de um texto cuja autoria é responsabilidade dos atores da cena didática, professor e alunos. Para tal, interrogamos esses discursos da sala de aula sob uma tríplice ótica: em primeiro lugar, a oposição que se verifica entre trabalho coletivo e trabalbo individual; a seguir, a dupla função exercida pela linguagem no referido contexto, a saber, a linguagem enquanto trabalho e a linguagem sobre o trabalho; finalmente, a distância que se verifica entre o trabalho prescrito e o trabalho real da sala de aula.

PaLAVRas-CHave: Discursos da sala de aula; Enunciação; Trabalho.

D.E.L.T.A., 19:1, 2003 (155-180) 
RÉSUMÉ: Cet article est centré sur l'analyse des discours de la classe de langue étrangère dans une situation particulière: l'enseignement du français comme langue professionnelle. Notre but sera de dégager des marques énonciatives qui contribuent à cerner la nature du travail réalisé en classe à travers la construction collective d'un texte entreprise par les acteurs de la scène didactique, enseignant et apprenants. Pour ce faire, nous interrogeons ces discours de la classe sous une triple optique: tout d'abord, l'opposion qui se vérifie entre travail collectif $X$ travail individuel; ensuite, la double fonction exercée par le langage dans un tel contexte, à savoir langage en tant que travail $X$ langage sur le travail; finalement, la distance qui se creuse entre travail prescrit $X$ travail réel.

MoTS-CLÉs: Discours de la classe; Énonciation; Travail.

\section{Introduction}

L'intérêt manifesté récemment par nombre de chercheurs sur le rôle joué par les activités de langage dans la compréhension du monde du travail a été la motivation centrale de cette approche des discours de la classe de langue étrangère.

S'attaquer au rôle du langage dans des situations de travail soulève des questions dont les enjeux sont très variés. D’une part il est question d'évaluer les moyens dont on dispose pour répondre à une demande sociale, comme par exemple lorsqu'il s'agit de contribuer à l'amélioration d'un poste de travail ou d'une relation de service, à la formation professionnelle ou à la mise au point d'une nouvelle technologie, entre autres. D'autre part on se voit confronté à un enjeu théorique: étant donné la complexité du monde du travail, toute investigation présuppose l'action coordonnée de plusieurs disciplines, la linguistique devant s'ouvrir à d'autres savoirs et à la collaboration avec des chercheurs travaillant dans des domaines variés. Ce n'est jamais une entreprise solitaire car, en effet, le travail nous concerne tous et ne peut pas constituer la chasse gardée d'un spécialiste quelconque. Aussi est-ce l'occasion pour le linguiste de mettre à l'épreuve les connaissances qu'il a accumulées pour pouvoir juger de leur efficacité et de la pertinence des méthodes qu'il a sous la main.

En ce qui concerne la place occupée par le langage dans son rapport aux situations de travail, l'organisation scolaire ne saurait constituer une exception à la règle et nous offre elle aussi un large éventail de pratiques 
langagières: les réunions du corps enseignant, les contacts avec les parents, le travail en classe, les échanges verbaux dans les couloirs, à la récréation, etc. Comme toute organisation de travail, l'école est elle aussi une "usine à mots" ${ }^{1}$. Plus que cela, il serait tout à fait légitime d'affirmer qu'elle est le lieu par excellence de l'exercice langagier: le travail qui s'y accomplit (quel que soit son but majeur - la socialisation, l'instruction, l'évaluation des compétences, le développement d'une performance) se trouve dans une étroite dépendance vis-à-vis de l'activité langagière qui lui sert de support.

Dans cet article nous centrerons l'attention sur les discours de la classe dans un contexte particulier: l'enseignement du français comme langue professionnelle. L'intérêt porté à cette situation d'enseignement a conduit les membres de l'équipe "Discours d'enseignement et interactions" ${ }^{2}$ vers le choix d'un corpus collectif de travail qui a été l'objet de multiples approches au cours des années 1997/98. Dans notre cas nous nous sommes intéressé précisément à la nature du travail qui est fait en classe à travers l'analyse de fragments extraits de deux sources différentes:

- situation d'enseignement A - transcription d'un cours de français des affaires à l'Alliance Française à Paris en octobre 1996, réunissant des étudiants d'origine diverse, niveau moyen;

- situation d'enseignement B - transcription d'un cours de français commercial à l'Institut Catholique de Paris en novembre 1995, réunissant aussi des étudiants d'origine diverse, niveau avancé.

En classe, on a un travail qui s'accomplit quasi exclusivement par les activités de langage - nous disons bien quasi exclusivement car il ne faut tout de même pas négliger la place occupée dans l'espace-classe par les gestes, le regard, les mouvements, les activités physiques les plus variées. Sous ce rapport, la singularité du travail réalisé en classe semble précisément résulter de l'intrication de ces différents plans d'activités qui constituent autant de pratiques sociales langagières et non langagières.

Pour justifier le point de vue qui est le nôtre ici, nous considérons utile d'introduire préalablement une réflexion concernant une double question:

\footnotetext{
Nous empruntons l'expression à Boutet, Gardin \& Lacoste (1995).

Equipe coordonnée par F. Cicurel au Cediscor, Université Paris 3, Sorbonne nouvelle.
} 
d'abord, ce qui nous autorise à parler de l'espace-classe en tant que lieu de travail; ensuite, à qui nous attribuons la responsabilité de ce travail.

\section{Travaille-t-on en classe? Qui est-ce qui travaille?}

Pour une approche des discours de la classe en tant que lieu de travail, nous nous heurtons à des difficultés de différents ordres:

- tout d'abord, le prestige acquis par d'autres approches des discours de la classe qui depuis les années 60 ont fait l'unanimité des travaux en linguistique appliquée, à savoir l'étude scientifique des principes et de la pratique de l'enseignement-apprentissage de langues (étrangères) ${ }^{3}$;

- ensuite, la difficulté qui consiste à définir ce qu'on appelle travail, si l'on considère que cette notion ne pourrait se contenter d'aucune définition ramenant à l'une de ses manifestations particulières (le travail esclave, le travail salarié, par exemple);

- troisièmement, le besoin d'avancer un argument qui puisse étayer l'idée que nous avons de la classe et qui nous autorise à parler spécifiquement de ce travail réalisé en classe.

Vis-à-vis de ces difficultés, nous assumons de notre part que, faute d'une définition du terme qui soit suffisamment souple pour accueillir le vaste éventail de modes d'actualisation de ce qu'on désigne travail, nous élargirons le sens de ce terme, ne serait-ce que provisoirement, à toute forme d'action humaine située dans le temps et dans l'espace susceptible de produire une transformation sur l'environnement.

Cette définition de travail est certes insuffisante, mais elle a au moins le mérite de mettre le doigt sur une question qui nous paraît incontournable et pourtant souvent négligée ${ }^{4}$ : le besoin d'une réflexion, si minime soitelle, sur le concept de travail. Dans notre cas, cette réflexion nous paraît

3 Nous renvoyons le lecteur à Celani 1992.

4 Nous croyons pouvoir repérer un signe de cette négligence dans le silence gardé dans plusieurs ouvrages sur le terme travail, comme c'est le cas par exemple du Vocabulaire de l'Ergonomie de Montmollin (1995), dont le vaste éventail d'entrées n'inclut pourtant pas celle de travail; on y trouvera tout au plus l'entrée travail posté. 
d'autant plus nécessaire que nous nous proposons de traiter la classe de langue étrangère justement en tant que ... lieu de travail!

Nous voudrions quand même retenir la leçon de Schwartz (1992) lorsque l'auteur dénonce l'anhistoricisme et l'essentialisme de toute réflexion sur le concept de travail qui ne problématiserait pas la commensurabilité de phénomènes situés en des périodes historiques et en des lieux géographiques distants. Autant dire que LE travail dans l'abstrait, cela n'existe pas.

Pour pallier autant que possible l'insuffisance de notre définition de travail, nous rappelons ici la thèse de Schwartz (1997) selon laquelle, pour faire vite, tout travail humain peut se définir comme le lieu d'une dialectique entre un premier registre (celui des antécédents normant et anticipant l'activité) et un second registre (la gestion des dimensions singulières de la situation $)^{5}$.

Nous voudrions profiter de la leçon de l'auteur concernant la distance entre ces deux registres (celui des normes antécédant une activité et celui de la singularité de toute activité) pour traiter de la distance qui se vérifie entre les discours sur le travail pédagogique (qui, d'une certaine façon, anticipent sur les fonctions exercées par l'école) et les pratiques pédagogiques qui s'actualisent dans un tel contexte (des pratiques qui ne coïncident pas avec ce qui s'affiche dans de tels discours).

Nombre d'auteurs ont souligné ce que Enguita (1989:137) appelle "la force de l'idéalisme dans le champ du discours scolaire". Selon l'auteur, on dirait que l'histoire pédagogique n'a pas contribué à déceler ni à comprendre la vraie histoire de l'éducation, car on a trop insisté sur "la noblesse des contenus" de l'éducation et trop peu sur "la réalité de la forme" de ces contenus. Autrement dit, il aurait fallu débusquer, sous cette fameuse transmission de savoirs, la force disciplinaire qui a toujours rythmé le travail à l'école depuis l'aube des premiers systèmes scolaires, lesquels répondaient à des impératifs d'ordre politique, religieux et militaire, jusqu'à la réinvention de l'école dans le cadre des relations de production

5 Notre intérêt pour ce double registre dont parle l'auteur ne signifie pas que l'approche que nous revendiquons ici corresponde aux préconisations méthodologiques des investigations des situations de travail, étant donné que nous travaillons sur des corpus rassemblés avant notre intervention. 
capitalistes $^{6}$. Nous n'insisterons pas là-dessus; nous nous contenterons d'emprunter la réflexion de Althusser rappelée par Enguita (1989) selon laquelle on apprend à l'école, certes, à lire, à écrire, à faire des calculs, on y accède à une "culture scientifique" ou "littéraire", mais en même temps on y apprend "les règles du 'comportement bienséant', c'est-à-dire, les règles de l'attitude convenable à observer selon le poste 'destiné' à tout agent de la division du travail: règles de morale, de conscience civique et professionnelle ..." (Enguita, 1989:147).

Pour Enguita (1989), si l'on met l'accent sur les contenus de l'éducation au détriment du débat concernant les pratiques scolaires qui soutiennent ces contenus, c'est parmi d'autres raisons parce qu'il est beaucoup plus valorisant pour l'enseignant de se croire responsable de la transmission (ou construction) de savoirs que de se voir attribuer le rôle d'agent de formation des mentalités dociles et disciplinées nécessaires à l'exercice d'une forme quelconque de pouvoir. En effet, "la socialisation en famille pouvait être adéquate et suffisante pour préparer le paysan à accepter les rapports de dépendance personnelle vis-à-vis du seigneur féodal, mais ne le serait pas pour garantir l'insertion dans l'organisation impersonnelle et réglementée de l'entreprise moderne. C'est là justement qu'intervient l'école." (Enguita, 1989:161).

Sur le même ton, Guattari (1981) explicite cette inévitable parenté entre ce qu'on fait à l'école et ce qu'on fait à l'usine:

L'école primaire à l'époque de Jules Ferry mettait en jeu des sortes de rites de passage vers des périodes encore tardives de la vie de l'enfant par rapport à ceux que nous connaissons maintenant; à l'école primaire, l'enseignant tenait avant tout à initier les enfants à un certain type de loi, de discipline; il leur apprenait à faire la queue, à ne parler que lorsqu'on le leur demandait, etc... Ce genre d'école correspondait à un certain mode d'organisation de la production, par exemple celui des manufactures, du travail à la chaîne, etc..., et de l'organisation militaire de "masse". (Guattari, 1981:52)

Étant donné le lien étroit qui s'établit historiquement entre l'école et le monde du travail - un lien que nous avons à peine évoqué -, nous nous

6 Voir à ce sujet le minutieux parcours de l'institution scolaire présenté par Enguita (1989) depuis le Bas Empire romain jusqu'à l'avènement du capitalisme. 
permettrons de parler dans cet article non seulement du travail entrepris par l'enseignant, mais aussi du travail réalisé par le groupe d'apprenants en classe. Ici la réflexion de Lapassade (1998) concernant l'apprentissage du "métier d'élève" pourra peut-être confirmer notre point de vue:

L'élève qui arrive à l'école doit commencer par apprendre 'le métier d'élève'; c'est un moment essentiel dans une carrière d'élève, un premier passage sur le chemin de la vie scolaire. Un second passage - une 'transition traumatique' - c'est celui de l'école primaire à l'école secondaire, etc. (Lapassade, 1998:42)

Outre les arguments que nous venons de présenter concernant l'institution scolaire en tant que formatrice du futur travailleur, nous rappelons aussi que:

- le fait que cette modalité de travail (le travail entrepris par les apprenants) ne soit pas rémunérée ne nous semble pas constituer une raison suffisante pour effacer l'activité industrieuse exercée par les apprenants en classe et la part qu'elle joue dans l'actualisation de ce qu'on appelle "le travail de la classe";

- le travail entrepris par l'enseignant ${ }^{7}$ nous semble avoir ceci de particulier qu'il n'est visible ni faisable qu'à travers la co-participation de cet acteur de l'espace-classe qu'est l'apprenant: le travail de l'enseignant ne saurait être décrit comme une "offre de service" exigée par la société à l'intention des apprenants, puisqu'il n'a pas lieu indépendemment de ce que peuvent faire - et de ce que font effectivement - ceux-ci;

- à l'école, l'apprenant est confronté à un même genre de motivations extrinsèques que l'on retrouve dans le monde du travail, qui sont autant de contreparties qui découlent de l'aliénation du travailleur par rapport au processus et au produit de son travail: approbation sociale, opportunités de promotion, possibilité d'éviter des sanctions, etc;

- en tant que linguiste travaillant dans le cadre d'une approche discursive, si l'on veut appréhender le travail des enseignants par le biais

\footnotetext{
7 Lorsque nous parlons de travail de l'enseignant ici, nous nous référons au travail réalisé en classe, et non pas à d'autres formes d'interventions censées être de la compétence de l'enseignant telles que la participation aux conseils de classe, la présence à des réunions de différents types, la préparation des cours, etc.
} 
de sa dimension langagière, il serait paradoxal de prétendre déconnecter ce que font ces deux agents de la scène didactique (enseignant et apprenants): le principe dialogique et le primat de l'interdiscours ne le permettraient pas.

Réduire ce travail assuré par l'apprenant - un travail qui serait le garant même du travail de l'enseignant - à la condition de "non travail" reviendrait à gommer ce lien qui donne son ton à l'institution scolaire telle que nous la connaissons aujourd'hui. En effet, une approche qui nous parait tout à fait pertinente serait celle qui pourrait accueillir cette dimension "laborieuse" de ce que font les apprenants à l'école en reconnaissant que ce qu'ils font c'est bel et bien ... travailler! Un travail qui serait bien entendu à la mesure de leur âge et de la part de "labeur" qui leur est impartie en société.

Une telle conception élargie de travail se justifie à plus forte raison dans l'actualité, si l'on en croit le témoignage de Guattari:

... la formation du travailleur et du soldat implique de nos jours une intégration maximale dans les processus de sémiotisation scripturaux. Devant le poste de télévision, les enfants "travaillent", de même qu'ils "travaillent” à la crèche, avec des techniques de jeu qui sont conçues pour améliorer leurs performances perceptives. D'une certaine manière on peut même considérer que ce travail est comparable à celui des apprentis à l'école professionnelle, ou bien à celui des ouvriers métallurgiques qui se recyclent pour s'adapter à de nouveaux types de travail à la chaîne. Il ne serait pas concevable dans la société actuelle que l'on puisse former un travailleur sans cette préparation qui se fait en famille, à la crèche, avant même l'entrée à l'école primaire. (Guattari, 1981:52)

Au bout de toutes ces réflexions qui devront nous permettre de mieux cerner les liens qui se vérifient entre l'école et le monde du travail - des réflexions motivées par le besoin de justifier ce qui nous autorise à parler du travail réalisé par l'enseignant et par l'apprenant -, nous voudrions rappeler que, si Boutet (1993) cite des chercheurs issus de différents domaines (des ergonomes, des sociologues, des spécialistes des sciences de la gestion, des philosophes et aussi des linguistes) qui "ont contribué à la description d'une part souvent mal connue de l'activité de travail, la part des échanges et des productions symboliques", nous, à notre tour, nous voudrions ici contribuer, compte tenu des limitations que nous avons déjà mentionnées, à la description d'une part souvent mal connue des échanges 
verbaux en classe: la part du travail qui s'y produit. Pour ce faire, nous proposons d'interroger ces discours de la classe sous une triple optique: tout d'abord, la nature du travail qui se réalise dans cet espace (travail collectif x travail individuel); ensuite la fonction exercée par le langage (langage en tant que travail x langage sur le travail); finalement, le double visage du travail (le prescrit $\mathrm{x}$ le réel) et les voies d'investigation qui s'ouvrent à travers une approche discursive de la question.

\section{Travail en équipe $\mathrm{x}$ travail solitaire}

Quelle est la logique qui sous-tend les échanges verbaux en classe de langue? A quels moments précis l'enseignant partage-t-il la scène didactique avec l'apprenant ou alors, à l'inverse, décide-t-il de garder le tour pour engager de longues tirades?

La question que nous nous posons ici concerne directement la place occupée par le langage dans le travail réalisé en classe de langue: s'agit-il d'un travail en équipe auquel participent enseignant et apprenants ou d'un travail plutôt monologal?

Nous soutiendrons que l'unique réponse possible à ces questions consiste à voir dans la classe de langue le lieu d'un équilibre très délicat entre pratiques monologales et pratiques dialogales. Nous introduirons le débat par l'investigation des conditions sous lesquelles enseignant et apprenants prennent en main le travail à réaliser en classe.

"En classe, on ne dit pas à autrui des choses qu'on sait déjà avant que celui-ci ne les énonce". Cette reformulation du texte de Boutet explicite l'un des traits les plus saillants de la classe de langue en tant que lieu de travail: l'enseignant doit faire en sorte que l'apprenant dise ce que l'enseignant espère, sous les formes les plus diversifiées et les plus inattendues qui soient. Cela a un rapport direct avec la demande de dire trait constitutif des discours de la classe - et aussi avec un certain piétinement de la communication en classe $^{8}$ : le travail fait en classe se déroule sur un rythme plus ou moins ralenti car il faut avant tout susciter

8 Pour la description du rituel communicatif dans les échanges d'enseignement, voir Cicurel 1990. 
des échanges, tenir en suspens l'information que l'enseignant connaît déjà pour la faire découvrir aux apprenants. Passons à l'analyse d'un fragment qui illustre ce que nous venons de dire.

Fragment $\mathrm{n}^{\circ} 1$ - Situation d'enseignement A; enjeu didactique: découverte avec le groupe d'apprenants d'un concept précédemment travaillé en classe (le concept de chiffre d'affaire consolidé)

P: (...) CHAQUE filiale a son chiffre d'affaire et après on fait le chiffre d'affaire de TOU-TES les filiales + ça s'appelle comment d'ailleurs? + le chiffre d'affaire de toutes les filiales?

Af: hum

P: c'est loin ça hein + c'est très loin ++ vous vous souvenez? + hein

As: (silence puis rires)

P: c'était dans l'exercice lacunaire sur les petites annonces + il fallait compléter + vous savez ce fameux compta:ble expert comptable + vous l'avez là? + vous pouvez retrouver le nom qui convient +++ hein lorsque l'on comptabilise le chiffre d'affaires de toutes les filiales + on a le chiffre d'affaire du groupe + comment ça s'appelle le chiffre d'affaire du groupe? + hein le bénéfice du groupe ++++ (bruits de papiers tournés) c'était un exercice lacunaire ++ pour le comptable les arrêtes mensuels + les bilans +++ (bruits de page) allez + qui trouve le mot?

(...)

Af: le budget

P: ah non le budget c'est autre chose Natacha (...)

(longue digression pour expliquer le sens du mot budget)

P: (...) bon alors c'est pas le budget d'une entreprise + hein vous avez trouvé ce mot ou pas?

As: non XXX

P: non?

Af: XXX consolidé

P: ah merci + ouf ++ ouf + CONSOlidé $(. .$.$) le chiffre d'affaires consolidé (. .$.

La démarche adoptée par l'enseignant qui avait pour but le rappel d'un concept déjà travaillé en classe consiste à fournir des pistes de différents ordres jusqu'à ce que le concept soit trouvé. Si nous parlons ici de piétinement de la communication ou ralentissement du travail de la classe c'est parce que l'enseignant refuse de donner tout court la bonne réponse et attend que les apprenants puissent la trouver à travers des pistes variées, même si pour cela il faut "perdre du temps". Dans le fragment cité, les 
acteurs de la scène didactique mettent trente tours de parole pour arriver à la découverte du concept souhaité!

Il y a des moments où, différemment de ce que nous venons de voir, une question ou alors une demande d'achèvement peuvent constituer des stratégies qui ont pour effet d'accélérer le rythme de travail de la classe:

Fragment $\mathrm{n}^{\mathrm{o}} 2$ - Situation d'enseignement B; enjeu didactique: compréhension de l'expression faire face à quelque chose

P: qu'est-ce que ça veut dire faire face à la concurrence?

Af: ++ être capable

P: oui : être capable de vendre nos produits même s'il y a des ... concurrents hein donc il faut être compé...

Af: titif

P: titifs merci beaucoup (elle écrit au tableau) nous devons être compé-titifs (...)

La question lancée par l'enseignant ("qu'est-ce que ça veut dire faire face à la concurrence?") est loin de ralentir le rythme du travail en classe: face à la réponse de l'apprenant qui essaie de reformuler l'expression faire face à la concurrence en disant "être capable", l'enseignant reprend la parole et anticipe l'explication complète que l'apprenant n'aurait peut-être pas formulée ("être capable de vendre nos produits même s'il y a des ... concurrents hein”). Dans des cas pareils, l'action précipitée de l'enseignant se trouve justifiée par le fait que celui-ci prend prétexte d'une bribe de réponse à peine ébauchée par l'apprenant. Il se peut aussi que l'enseignant ait recours à la demande d'achèvement, comme on le voit dans "il faut être compé..." - stratégie qui lui permet de "gagner du temps" (l'apprenant n'a qu'à compléter le mot attendu), tout en gardant la dimension interactive du travail en classe.

Qu'il s'agisse de ralentissement ou accélération du temps dont on dispose en classe, ce qui nous paraît finalement important ici c'est la place accordée à cette présence de l'apprenant dans son rapport avec le travail réalisé par l'enseignant.

Si d'une part l'enseignant tient à partager la scène didactique avec le groupe d'apprenants (il refuse dans la majorité des cas de faire étalage de son savoir), d'autre part il y a des moments où tous les espaces sont saturés de sa présence, à l'instar du fragment que nous reproduisons ci-dessous: 
Fragment $\mathrm{n}^{0} 3$ - Situation d'enseignement B; enjeu didactique: découverte de l'expression protection sociale.

P: payer sa participation voilà à à aux frais généraux de tous les gens du pays c'est pour ça qu'on appelle tout ça + la pro-tec-tion (elle écrit au tableau) quel est le deuxième mot après protection $++\mathrm{i}$ faut un adjectif quel adjectif? + ça vous avez besoin de ça pour l'examen + hein la protection + on en parle tous les jours tous les jours à la radio: à la télévision + la protection Sociale (elle écrit au tableau)

Les différentes activités ${ }^{9}$ assurées par l'enseignant peuvent être schématiquement résumées de la sorte:

- assertion (“c'est pour ça qu'on appelle tout ça + la pro-tec-tion”)

- demande de complémentation de l'asserté ("quel est le deuxième mot après protection")

- piste d'ordre métalinguistique (“i faut un adjectif quel adjectif?”)

- justification de la demande faite ("ça vous avez besoin de ça pour l'examen + hein")

- piste d'ordre pragmatique renvoyant au vécu des apprenants (“on en parle tous les jours tous les jours à la radio: à la télévision”)

- réponse ("la protection Sociale”)

Situation quelque peu paradoxale du travail qui se fait en classe dans la mesure où deux attitudes centrales semblent s'alterner: un travail "à la chaîne”, collectif (dans lequel l'enseignant est relayé par ses "pairs" - les apprenants - dans la co-construction du savoir, ne serait-ce que sous le mode de la simulation) et un travail plutôt "artisanal" et solitaire (dans le sens où l'enseignant assurerait l'exécution de toutes les étapes prévues comme nécessaires à la compréhension d'un concept, par exemple).

\footnotetext{
9 Comme on l'a déjà dit, puisque nous travaillons sur des corpus rassemblés avant notre intervention, le concept d'activité auquel nous avons recours ne peut pas rendre compte de toutes les possibilités dont nous parle Faïta (1995a:86): “Analysant la 'matérialité langagière' des échanges, il [l'intervenant] se trouve en position de la dépasser pour tenter d'éclairer, à partir de ce que lui enseignent les rapports entre ce qui se dit, ce qui ne se dit pas, ce qui aurait pu se dire autrement, les zones d'ombre ou 'méandres' recelant cette part de l'activité qui échappe nécessairement au codage."
} 


\section{Langage en tant que travail $x$ langage sur le travail}

Dans le genre d'enseignement qui nous concerne ici, à savoir, l'enseignement du français comme langue professionnelle à l'intention d'apprenants étrangers, l'enseignant doit certes accorder une importance fondamentale à la langue: pour que l'apprenant puisse entrer dans le monde du travail, pour qu' il puisse y trouver sa place, il doit maîtriser la languecible. C'est à ce niveau que nous pouvons saisir le rôle des pratiques de langage en tant que travail: en classe de langue, on se doit de construire une compétence linguistique. Comme le remarque Boutet, "l'activité de langage suppose nécessairement la dimension de la langue, entendue comme un système de signes et une organisation particulière de ces signes" (Boutet 1993:113). A ce qu'il semble, le grand défi qu'il faut relever dans ce contexte particulier de travail qu'est la classe de langue réside précisément dans la construction d'un système extérieur aux individus qui leur permettra de mouler le sens de leurs interactions quotidiennes.

Dans le corpus sur lequel nous avons travaillé, la compétentialisation du locuteur non natif est visible lorsque, parallèlement à l'introduction d'un vocabulaire qui a un usage circonscrit au domaine de spécialité du français commercial, l'enseignant insiste sur des mots ou expressions qui auraient libre cours en langue maternelle. Il est facile d'apercevoir que le travail sur des expressions telles que salaire net imposable, points de vente, rentrer des devises, toutes taxes comprises se justifie par l'objectif même de l'apprentissage du français commercial (domaine d'activité qui suppose une pratique discursive spécifique), tandis que toucher un salaire, toucher une personne, à l'amiable sont autant d'expressions qui circulent dans les échanges verbaux quotidiens et qui sont maîtrisées par un locuteur natif sans aucun entraînement préalable spécifique.

En ce qui concerne la compétentialisation du groupe d'apprenants, il y a au moins deux situations dignes de mention: en premier lieu, la compétentialisation de ce locuteur non natif qui n'aurait pas encore la maîtrise d'un vocabulaire non spécialisé, cas de figure que nous illustrons dans le fragment $\mathrm{n}^{\mathrm{O}} 4$; ensuite, la compétentialisation au niveau des connaissances grammaticales de la langue-cible, à l'exemple des fragments 5 à 7. 
Fragment $\mathrm{n}^{\mathrm{O}} 4$ - Situation d'enseignement B; enjeu didactique: élucidation du sens de l'expression ne pas en voir la couleur

P: (...) les gens COtisent parce que les gens PAYENT i payent quoi leur contribution leur participation alors je répète ici vous avez le salaire le salaire... brut + ici vous avez tous les toutes les comment est-ce qu'on les appe:lle + toutes ces choses.....

Af: XXX

P: oui les Retenues + les Retenues + ça veut dire que tu n'en vois pas la couleur + qu'est-ce que ça veut dire tu n'en vois pas la couleur + eh ben elle a compris qu'estce que ça veut dire tu n'en vois pas la couleur ça passe sous ton nez, comme ça euh zioup tu vois en haut + tu vois un joli chiffre et puis tu regardes en bas $\mathrm{hm} \mathrm{hm} \mathrm{tu}$ n'en vois pas la couleur ce sont les re:tenues (...)

A remarquer que, s'il est certain que l'expression "ne pas en voir la couleur" n'est pas exclusive du domaine de spécialité concerné en classe (en l'occurrence, un cours de français commercial), il n'en reste pas moins que l'enseignant garde ce contexte de spécialité pour donner l'explication nécessaire ("ça passe sous ton nez, comme ça euh zioup tu vois en haut + tu vois un joli chiffre et puis tu regardes en bas hm hm tu n'en vois pas la couleur"). A vrai dire, il n'y a pas d'explication: ce que fait l'enseignant c'est contextualiser l'expression (on peut aussi supposer que le gestuel y est pour beaucoup!).

Pour ce qui est de la compétentialisation du locuteur en langue-cible au niveau grammatical, passons au fragment suivant:

Fragment $\mathrm{n}^{\circ} 5$ - Situation d'enseignement B; enjeu didactique: introduction d'une règle grammaticale (l'expression dans le cas où suivie d'un verbe au conditionnel)

$\mathrm{P}:(\ldots)$ dans le cas où un article est défectueux maintenant on peut dire dans le cas + est + défectueux mais autrefois qu'est-ce qu'on mettait à la place de dans le cas où un article EST qu'est-ce qu'on mettait en grammai:re ++ autrefois et même maintenant + quand on respecte

\section{A: XXX}

P: serait serait il faut le conditionnel hein dans: le cas où euh un article seRAIT défectueux hein + alors on met le conditionnel mais: actuellement on peut mettre le présent d'accord?

L'extrait ci-dessus témoigne de la nature du travail que l'on fait en classe de langue étrangère qui consiste précisément à compétentialiser le locuteur en langue-cible (en l'occurrence, emploi d'une forme verbale au conditionnel). Il serait légitime de voir dans ce que nous disons ici une 
sorte de tautologie: on est en classe de langue pour apprendre cette langue. Nous voudrions toutefois mettre l'accent non seulement sur cette activité qui se présente en tant que travail effectivement réalisé mais sur le mode dont s'actualise ce travail. En effet, lorsqu'on se penche sur la singularité des échanges dans ce contexte, on est contraint d'admettre que ce qui pourrait de prime abord paraître une réflexion tautologique n'en est pas une. Passons au fragment suivant:

Fragment $n^{\circ} 6$ - Situation d'enseignement B; enjeu didactique: contribuer à la découverte de modes variés d'expression en langue-cible (activité de paraphrasage)

P: (...) tous ces mots sont importants + bien sûr + ça fait RENTRER des devises + des devises de la MONNAIE étrangère + vous connaissez ce mot et A l'exportation ça veut dire euh SI l'on exporte + QUAND on exporte + au moMENT où l'on exporte + et bien les prix sont hors taxe (...)

A travers la lecture de ce fragment, on constate que le travail de compétentialisation qui se réalise en classe a ceci de particulier qu'il semble être nuancé d'une activité de reformulation qui frôle l'obsession. Dans le segment "SI l'on exporte + QUAND on exporte + au moMENT où l'on exporte", on se voit confronté à un dire qui se reprend, qui ne cesse de se reformuler, bref une énonciation singulière qui est le lieu d'une rencontre inouie (la langue et le discours). Ce discours saturé de langue dont témoigne l'insistance sur des modes variés d'expression nous fait penser à ce que nous avons décrit ailleurs comme "avalanche grammaticale" 10 .

Un cas limite du travail de compétentialisation du locuteur en languecible peut être illustré lorsque l'apprenant ne dispose pas des moyens linguistiques qui lui permettraient de s'exprimer en langue étrangère:

Fragment $\mathrm{n}^{0} 7$ - Situation d'enseignement A; enjeu didactique: explication du sens de l'expression ressources humaines

P: hein + alors qu'est-ce que ça peut vouloir dire ressources humaines? Expliquezmoi? Moi je ne sais pas ce que ça veut dire

Af: *contractation* de + c'est ça de personnel

P: de quoi? J'ai pas entendu le premier mot c'est peut-être bien + mais je ne l'ai pas entendu ++ vous pouvez répéter?

10 Voir Rocha 1996, "La fiction dans le cadre de l'interaction didactique: une lecture du théâtre de Ionesco". 
Af: ah je ne suis pas sûre (rire)

$\mathrm{P}$ : ça ne fait rien + qu'est-ce que vous comprenez de XXX

Af: *contractation* c'est ça?

P: de contractation + c'est pas français ça +++ qui peut me dire + ressources humaines + quel est le travail des ressources humaines +++ Fabien

Am: le recrutement

$\mathrm{P}$ : le recrutement hein + c'est ça voilà + c'est le recrutement $(. .$.

Ici nous assistons à un double travail de compétentialisation linguistique: non seulement celui de l'enseignant qui relève la faute commise ("contractation") et incite le groupe à la découverte du mot convenable ("recrutement"), mais aussi celui de l'apprenant qui, ne connaissant pas d'avance le mot en langue-cible, prend le risque d'inventer le mot souhaité - travail qu'il exécute ayant comme point de repère un radical qu'il puise de toute évidence dans sa propre langue maternelle ("contracter") suivi du suffixe nominalisateur en langue-cible (-ation).

Par ailleurs, il faut aussi familiariser l'apprenant avec l'univers de travail qui constitue à la limite la raison d'être de ce genre d'enseignement. Les discours tenus en classe doivent contribuer à la compréhension de ce monde du travail. Autrement dit, une marque constitutive de ces discours réside dans leur capacité de parler de cet univers, dans la possibilité qu'ils offrent d'anticiper ce monde du travail dont il est question - des pratiques de langage sur le travail.

Pour venir à bout de cette anticipation, la classe opère une mise en scène de ce monde du travail. Dans notre corpus, on remarque que c'est à travers l'acte de lecture que les acteurs de l'espace-classe récupèrent le travail de ceux qui ont besoin d'être compétitifs, qui multiplient leurs points de vente, qui se livrent à des pratiques de vente par correspondance, etc. Le fragment suivant en est très illustratif:

Fragment $n^{\circ} 8$ - Situation d'enseignement B; enjeu didactique: explication des stratégies commerciales adoptées par les entreprises pour être compétitives

$\mathrm{P}$ : voilà + donc + des mots importants + voilà + puisqu'ils veulent être tellement compétitifs + il faut qu'ils MULtiplient leurs points de vente + comment est-ce qu'ils multiplient leurs points de vente? Ils ont + une gamme de meubles pour les hypermarchés + ils ont + leurs propres magasins + et ils ont + une entreprise de $+\mathrm{v}$ p c tout ça hein? Qu'est-ce que ça veut dire v p c? + + la vente ..... par correspondance

Af: par correspondance 
Ici on parle à la 3ème personne du travail de ceux qui développent des pratiques commerciales. A travers une énonciation qui tient à distance cet univers des ventes, le groupe classe s'en distingue très nettement. Mais ce n'est pas toujours que l'identité du groupe classe est préservée et il y a des moments où les acteurs de la scène didactique se confondent avec les personnages de la fiction. On assiste alors à une sorte de mise en scène de l'énonciation originelle ${ }^{11}$ : les acteurs de la classe viennent habiter cet univers du travail. Pour illustrer cette énonciation, prenons connaissance du fragment suivant:

Fragment no 9 - Situation d'enseignement B; enjeu didactique: mise en place d'un contexte qui facilite la compréhension de l'expression faire face à quelque chose

P: ça vous le savez déjà + nous leur donnons + un fixe mensuel et euh une commission sur euh les ventes réalisées + donc ils ont un fixe mensuel et une commission sur les ventes vous le savez nous l'avons déjà vu + ENsuite ++ nous allons descendre tout en bas tout en bas de la première colonne + nous n'avons pas le monopole de la vente des meubles nous n'avons pas le monopole nous ne sommes pas les SEULS sur le marché en France d'accord nous continuons nous devons + être en mesure + estce qu'elle peut lire euh Jacqueline + tout en bas nous devons

Jacqueline: (elle lit) nous devons donc être en mesure de faire face à la concurrence

Le fragment cité ci-dessus met en relief une situation d'énonciation mixte et on assiste à la mise en scène d'un nous dont la référence ne cesse de glisser:

- tout d'abord, un nous qu'il serait légitime de faire coïncider avec les personnages de la fiction, c'est-à-dire les personnages du texte lu en classe: "nous leur donnons + un fixe mensuel et euh une commission sur euh les ventes";

- ensuite, un nous qui se réfere aux acteurs de l'espace-classe: "nous l'avons déjà vu + ENsuite ++ nous allons descendre tout en bas tout en bas de la première colonne";

- finalement un nous dont l'identité est plus floue et qui semble constituer une sorte de chevauchement des deux voix précédentes - les personnages de la fiction et les acteurs de la classe: "nous n'avons pas le

11 Voir à ce sujet la notion de glissando énonciatif (Cicurel 1996:86). 
monopole de la vente des meubles nous n'avons pas le monopole nous ne sommes pas les SEULS sur le marché en France d'accord nous continuons nous devons + être en mesure".

Double enjeu des discours tenus en classe de français comme langue professionnelle: compétentialiser l'apprenant en langue-cible et "former" le futur travailleur.

\section{Travail prescrit $\mathrm{x}$ travail réel}

Les concepts de travail prescrit et travail réel sont devenus classiques dans les études ergonomiques de langue française. Si nous évoquons ici ces deux concepts c'est parce que nous misons sur leur productivité pour la compréhension de la classe de langue en tant que lieu de travail.

Le travail prescrit, également désigné tâche, réfere à "tout ce qui, dans l'organisation du travail, définit le travail de chacun au sein d'une structure donnée" (Noulin 1992:32). Selon Noulin, le concept de travail prescrit recouvre un large éventail de facteurs: les objectifs à atteindre en contrepartie de la rémunération, la manière de les atteindre, les moyens techniques, la répartition des tâches, les conditions temporelles (les horaires, la durée) et sociales (qualification, salaire) du travail à être exécuté, les conditions de l'environnement physique. Bref, le concept de tâche renvoie au cadre formel qui précède la performance du sujet et qui exerce sur celui-ci un pouvoir coercitif.

Quant au travail réel ou activité, il s'agit de ce qui se produit effectivement lors de l'exécution de la tâche et qui n'est jamais simple réalisation de ce qu'on avait prévu. Aussi le travail réel comprend-il la manière dont les objectifs prescrits sont intériorisés par le sujet, le mode particulier dont celui-ci s'approprie les moyens techniques (y compris la mise en place d'un éventail plus ou moins large de moyens non prévus), les différentes représentations des sujets sur les conditions sociales qui définissent le travail, les imprévus de l'environnement physique, entre autres.

Les discours de la classe de français comme langue professionnelle sont très riches sous ce rapport-là. En tant qu'interaction finalisée se développant dans un lieu institutionnel déterminé, la classe nous permet tout abord d'appréhender ce qui relève du prescrit: 
- en ce qui concerne les moyens techniques, outre le tableau et le cahier de notes qui sont dans notre culture scolaire les lieux par excellence d'inscription du travail réalisé, le manuel pédagogique adopté pose des contraintes dans la mesure où il semble rythmer le travail de la classe ${ }^{12}$ ("nous allons descendre tout en bas tout en bas de la première colonne", "tout en haut hein de la page cinquante-trois première colonne");

- du choix de ce manuel découle aussi la scansion du temps de travail en classe: les différents chapitres figurant dans le programme font le balisage des moments où l'enseignant peut "reprendre le souffle" ("voilà ouf nous avons terminé ce chapitre sur les ventes");

- la nature du matériel présenté dans le manuel est déterminante de la nature du travail qui sera réalisé; conscient de la grande quantité de termes spécialisés introduits dans un même texte sur des activités commerciales, l'enseignant va centrer son travail sur l'étude du vocabulaire ("combien de mots nouveaux jusqu'à maintenant? + + y a des mots que nous avions déjà vus”, “qu'est-ce que c'est l'emballage? + Anieszka? + + encore un mot très important du de la feuille");

- cette présence massive de termes spécialisés relève d'une autre contrainte de ce genre d'enseignement: le recours à des documents authentiques. Dans les transcriptions sur lesquelles nous avons travaillé on se trouve confronté à des fragments où il est question d'un vrai cours de lecture - une lecture spécialisée, pourrions-nous dire, puisqu'il s'agit d'apprendre à lire par exemple des petites annonces (situation d'enseignement A) ou un bulletin de salaire (situation d'enseignement B). Comment justifier de tels choix? Est-ce qu'on sera dans l'avenir des destinataires ou émetteurs de ce genre de textes?;

- le soulagement de l'enseignant lorsque l'apprenant trouve la bonne réponse à une question formulée nous renvoie aux rôles assignés aux interactants en classe: il incombe à l'enseignant de transmettre des connaissances; quant aux apprenants, il ne suffit pas qu'ils retiennent les savoirs transmis, encore faut-il en donner des preuves;

- si l'apprenant est censé pouvoir donner des preuves de son apprentissage c'est parce qu'il y a une autre contrainte qui pèse lourd sur

12 Nous ne tiendrons pas compte ici de l'activité de préparation de cours entreprise par l'enseignant. 
le travail de la classe: l'évaluation des connaissances acquises. Ici on comprend la demande - très récurrente d'ailleurs - faite aux apprenants de retenir ce qu'ils ont déjà compris, d'apprendre ce qui leur est dit (commentaire de l'enseignant sur le mot consolidé dans l'expression chiffre d'affaires consolidé: "ça c'est un terme qu'il faut essayer d'apprendre hein", "ça vous avez besoin de ça pour l'examen hein");

- quant à la répartition des tâches et à la manière d'atteindre les objectifs de la classe, l'enseignant se doit de gérer les interactions: pour garantir un mode d'échanges collectif en classe, l'enseignant attire l'attention du groupe sur ce que dit un apprenant ("écoutez chut + écoutez bien ça écoutez son argumentation + écoutez ce qu'elle dit hein c'est intéressant"); pour rappeler à l'apprenant une des règles fondamentales de ce travail, l'enseignant n'hésite pas ("pourquoi est-ce que vous dites primaire? (...) parce que quand on dit quelque chose on le justifie d'accord”); ou alors à un apprenant qui ne parle pas assez fort ("bien fort qu'on vous entende hein);

- en ce qui concerne les conditions du travail réalisé en classe, on fait référence à un autre élément qui participe du cadre formel dans lequel a lieu l'interaction: le paiement des cours (puisqu'il s'agit de cours payants) assuré par les apprenants; ici on se voit confronté à ce qui constitue l'apprenant en tant que tel, c'est-à-dire ce qui le légitime dans la position d'apprenant au sein d'une organisation de travail et qui correspond à la contrepartie du salaire de l'enseignant. A titre d'illustration, nous rappelons le passage où l'enseignant explique la différence entre une société (qui poursuit des buts lucratifs) et une association (buts non lucratifs) et, pour faciliter la compréhension, cite l'Alliance Française comme exemple d'association à but non lucratif ("généralement ça vous fait sourire parce que vous me dites oh la la mais je paye mes cours à l'Alliance Française”).

Nous parlions plus haut de la distance qui se creuse inéluctablement entre travail prescrit et travail réel. Comment approcher cette distance à l'intérieur des situations de travail de la classe?

Pour introduire le débat, nous dirons qu'il y a toujours des écarts plus ou moins importants entre ce que l'enseignant compte faire en classe et ce qui se présente lors de la réalisation du cours. Comment appréhender ces écarts? Quelles pistes suivre? En guise de réponse anticipée à la question, nous dirons que cet écart se laisse apercevoir dans les digressions qui ont 
lieu au fur et à mesure que la classe avance, provoquées tantôt par une incompréhension, tantôt par un malentendu, etc. C'est ce qui se passe lorsque l'enseignant croit important d'interrompre momentanément le cours des événements prévus pour passer à autre chose dont la pertinence peut être mesurée avant tout par la participation des apprenants: un contenu non prévu peut être introduit comme réponse à une demande de l'apprenant. C'est à ce niveau-là que nous nous croyons autorisé à établir la distance entre le travail prescrit (les normes situées en amont de l'interaction) et le travail réel (ce qui est effectivement produit en classe).

Les malentendus constituent une deuxième entrée qui nous permet d'appréhender la distance qui sépare le travail prescrit et le réel qui s'actualise en classe. Dans notre corpus (situation d'enseignement A) l'apprenant prend la forme S.A. comme sigle de Siemens Automobile, alors que ça renvoie à la forme juridique de l'entreprise, société anonyme. comme le répète l'enseignant. Au bout de quelques tours de parole où s'alternent enseignant et apprenants, il y a prise de conscience et levée du malentendu. $\mathrm{Si}$ nous disons que le malentendu est une trace non équivoque du travail réel qui se fait en classe c'est parce qu'il n'est pas sans influence sur le déroulement postérieur de l'interaction didactique: l'enseignant va désormais redoubler d'attention quant aux occurrences de sigles figurant dans l'exercice qui fait l'objet du travail en cours ("tout le monde a trouvé G.S.F. là on a un sigle + là on a vraiment un sigle hein", "(SNCF) société nationale des chemins de fer hein là on a un sigle hein”).

\section{Conclusions}

Notre but dans ce travail était de problématiser la classe de langue sous le rapport de la nature du travail qui s'y réalise. Pour ce faire, nous avons accordé la priorité à trois questions qui nous ont paru centrales: premièrement, la nature du travail réalisé en classe, comprenant l'alternance d'activités monogérées et polygérées; ensuite, les différentes pratiques langagières qui s'y produisent (le langage en tant que travail et le langage sur le travail); finalement, la distance qui sépare le travail prescrit et le travail effectivement réalisé dans cet espace. En guise de conclusion, nous voudrions maintenant revenir sur chacune de ces trois questions pour en évaluer la portée et si possible avancer de nouvelles pistes de recherche. 
Comment se distribuent à l'intérieur de l'espace-classe les activités monogérées (lorsque l'enseignant prend le parti d'un travail plutôt solitaire) et les activités polygérées (lorsque prédomine le dialogal)? Voilà une question qui renvoit de toute évidence à l'un des paramètres du cadre formel qui définit l'espace-classe en tant que lieu de travail: la gestion du temps. Comme nous l'avons vu, l'option pour un mode d'action plus ou moins interactif en classe semble relever, ne serait-ce que partiellement, du temps dont on dispose pour mener à bout une activité quelconque. Il revient à l'enseignant de gérer ce temps de travail de la classe, qui semble osciller entre deux pôles - la dilatation et la contraction. Grosso modo cette répartition pourrait être définie de la sorte: du côté du temps qui se dilate et qui autorise l'enseignant à partager avec le groupe d'apprenants la tâche à accomplir, on assiste à des échanges verbaux qui font preuve de la coconstruction du savoir en classe ${ }^{13}$; du côté du temps qui se contracte, la transmission un tant soit peu hâtive d'un savoir assurée par l'enseignant tout seul. Bref tout se passe comme s'il fallait passer sans cesse d'un pôle à l'autre, de la simple transmission à la découverte collective (qu'il s'agisse de construction ou d'appropriation) d'un certain savoir, l'enseignant assurant continuellement le guidage de ce qui se déroule en classe.

Lorsque nous avons introduit le thème des pratiques langagières en tant que travail et sur le travail, nous avons parlé d'un double enjeu des discours tenus en classe de français comme langue professionnelle: la compétentialisation de l'apprenant en langue-cible et la "formation" du futur travailleur. Dans notre corpus ce travail de formation de ce futur travailleur semble être restreint à l'acquisition d'un lexique et de quelques tournures de phrase circulant dans un domaine discursif spécialisé, ce qui nous autorise de prime abord à avancer une première observation: la formation du futur travailleur est à tout le moins douteuse. Serait-il légitime d'en conclure que la compétentialisation du locuteur en langue-cible l'emporte sur la formation du futur travailleur dans un domaine quelconque? Serait-ce plutôt vers l'apprenant de la langue-cible que se

\footnotetext{
13 Il serait opportun de signaler ici que ce que nous considérons comme co-construction d'un savoir peut aussi correspondre à une sorte de découverte collective d'un savoir construit avant et ailleurs, c'est-à-dire simple appropriation de connaissances dont la classe ne peut pas revendiquer les droits d'auteur. C'est apparemment ce que nous avons pu observer dans notre corpus lorsque par exemple l'enseignant se doit de transmettre un nouveau lexique qui doit être mémorisé par l'apprenant.
} 
tournerait l'attention de la classe dans un tel contexte? Nous répondrons négativement à la question car le travail acharné sur ce lexique spécialisé et aussi la grande quantité d'informations à transmettre sur cet univers de travail spécifique font que les activités de langage entreprises par les apprenants se caractérisent quasi exclusivement par des productions monosyllabiques, au détriment de la formulation d'énoncés plus complets et de l'actualisation d'échanges verbaux authentiques. On se situe de toute évidence à mi-chemin entre les deux objectifs de formation, ce qui ne manque pas de constituer une situation quelque peu paradoxale: comment concilier ces deux objectifs de formation dans le travail réalisé en classe de langue étrangère comme langue professionnelle?

Par ailleurs, la mise en place de ce double objectif de formation joue également sur l'identité des acteurs de l'espace-classe: pour ce qui est de l'enseignant, celui-ci se doit de maîtriser autant que possible des connaissances relevant d'un domaine de spécialité qui n'est pas toujours le sien (l'enseignant n'est pas forcément un expert du domaine du tourisme ou des affaires commerciales); quant à l'apprenant, entre l'image du locuteur en langue-cible et celle du futur travailleur dans un domaine de spécialité quelconque s'interpose une troisième image, à savoir celle de l'apprenant qui devra donner des preuves du savoir acquis lorsqu'il faudra passer un examen. Nous sommes persuadé que ce "brouillage identitaire" donne son ton à la classe de langue étrangère comme langue professionnelle et détermine en partie le recours à certaines stratégies de bachotage d'un programme souvent très chargé.

Quant à l'opposition qui se vérifie entre le prescrit et le réel, nous rappelons que notre projet était tout simplement de pointer des pistes qui puissent nous permettre de saisir la singularité des échanges en classe par rapport à ce qui n'avait pas été préalablement prévu. En nous tournant vers notre corpus, nous avons discuté du rôle joué par les digressions et les malentendus en tant que manifestations de cette distance. Nous n'avons pas oublié de remarquer que ce n'étaient là que des pistes qui nous conduisaient vers ce décalage constitutif de tout acte de travail. A ce sujet, nous voudrions encore ajouter qu'il serait tout à fait souhaitable de relativiser la bipartition réel x prescrit, comme nous avertit Faïta (1995b: 428): “... comme dans toute manifestation de l'activité humaine, le comportement des acteurs, les séquences d'actes et des gestes qu'ils accomplissent, ne sont que la partie visible d'un réel qui déborde de toutes parts les limites perceptibles entre lesquelles ils se meuvent”. 
Un dernier point qu'il nous paraît important de souligner ici est le rôle joué par le document authentique dans le travail de la classe de langue étrangère. Quel est le statut du document authentique en classe de langue étrangère visant à une formation professionnelle et dans quel sens nous permet-il de mieux cerner la nature du travail qu'entreprennent enseignant et groupe d'apprenants dans un tel contexte?

Si on dit authentique, c'est parce qu'on sait que le document sur lequel on travaille a une vie "indépendante" de la classe, c'est-à-dire il a été produit à des fins non didactiques, bref il circule ailleurs. Il a une finalité autre que de servir de support méthodologique en classe de langue. En se l'appropriant, la classe en fait un outil de travail à d'autres fins: la classe va y prélever ce qui lui paraît le plus pertinent vis-à-vis de ses propres objectifs. C'est dans ce sens que nous rejoignons les propos de nombre de chercheurs qui, tournés vers les discours de la classe de langue, parlent de fictionnalisation du document authentique en classe.

Le recours à l'authentique nous fait découvrir quelques-uns des présupposés qui étayent la démarche de l'enseignant: faire plonger l'élève dans la réalité des échanges quotidiens du monde du travail; faire connaitre la culture du groupe au sein duquel le document authentique a été produit (le concept de culture comprenant les habitudes, les règles interactionnelles, etc.)

Par ailleurs, cette présence de l'authentique nous permet de débusquer une dimension stratégique de cette modalité d'enseignement: comment accrocher le public? En effet, on pourrait avancer l'hypothèse selon laquelle la classe s'empare du document authentique pour "redonner du souffle" à son travail: à défaut d'une méthode qui soit à même de susciter l'intérêt de la classe, l'authentique peut vraisemblablement constituer une stratégie pour capter l'apprenant des niveaux plus avancés. Autrement dit, l'espaceclasse se donne les moyens de mettre en place un travail plus "pratique", plus "vrai" - un travail susceptible d'intéresser les élèves qui, ayant conclu le cours de base de langue française et désireux de sortir du carcan didactique, sont potentiellement des candidats aux niveaux avancés. Par ce biais, la classe prend des airs "pratiques" pour gagner de l'intérêt auprès de son public ${ }^{14}$.

14 Le rôle stratégique de cette modalité d'enseignement d'une langue étrangère semble répondre à un besoin des responsables du travail pédagogique qui, coincés devant l'évasion progressive des 
Parler du travail qui se réalise en classe de langue étrangère alors que la classe se propose de parler du monde du travail (les activités reliées au tourisme ou aux affaires commerciales), voilà une démarche qui nous a permis d'approcher sous un jour particulier les discours qui circulent dans l'espace-classe et de soulever de nouvelles pistes de recherche. Autant de questions concernant le langage dans son rapport subjectif au travail sur un terrain qui pour la plupart reste encore vierge. Les paris sont ouverts.

E-mail: rochadm@uol.com.br

Recebido em abril de 2002

Aprovado em março de 2003

\section{RÉfÉRENCES Bibliographiques:}

Boutet, J. 1993. Activité de langage et activité de travail. Education permanente 116 (Comprendre le travail - première partie).

Boutet, J., Gardin, B., Lacoste, M. 1995. Discours en situation de travail. Langages 117. Paris: Larousse.

Celani, M. A. A. 1992. Afinal, o que é Lingüística Aplicada?. Paschoal, M. S. Z. de \& Celani, M. A. A. 1992. Lingüística Aplicada - da aplicação da Lingüística à Lingüústica transdisciplinar. São Paulo: Educ.

Cicurel, F. 1990. Eléments d'un rituel communicatif dans les situations d'enseignement. Dans Cicurel, F., Variations et rituels en classe de langue. Paris: Hatier.

. 1994. Marques et trraces de la position de l'autre dans les discours de l'enseignement des langues. Dans Les Carnets du CEDISCOR 2. Paris: Presses de la Sorbonne Nouvelle.

. 1996. L'instabilité énonciative en classe de langue: du statut didactique au statut fictionnel du discours. Dans Les Carnets $d u$ CEDISCOR 4. Paris: Presses de la Sorbonne Nouvelle.

Enguita, M.F. 1989. A face oculta da escola - Educação e Trabalho no Capitalismo. Porto Alegre: Artes Médicas.

élèves, essaient de répondre à une attente tout à fait légitime des étudiants: à quoi ça sert enfin d'étudier une langue étrangère? 
FAïTA, D. 1995a. Dialogue entre expert et opérateur: contribution à la connaissance de l'activité par l'analyse des pratiques langagières. Connexions $n^{\circ}$ 65. Ramonville Saint-Agne: Érès. . 1995b. Interaction verbale et gestion des variables du travail. In: D. VéroniQue; R. Vion (eds.) Des savoir-faire communicationnels, Publications de l'Université de Provence.

Guattari, F. 1981. Revolução molecular: pulsações políticas do desejo. São Paulo: Brasiliense.

Lapassade, G. 1998. Microsociologie de la vie scolaire. Paris: Economica.

Montmollin, M. de 1997. Vocabulaire de l'Ergonomie. Toulouse: Octares.

Noulin, M. 1992. Ergonomie. Paris: Techniplus.

Rocha, D. 1996. La fiction dans le cadre de l'interaction didactique: une lecture du théâtre de Ionesco. Dans Les Carnets du CEDISCOR 4. Paris: Presses de la Sorbonne Nouvelle.

SchWARTZ, Y. 1992. Sur le concept de travail. Colloque 'Travail: Recherches et Perspectives', miméo.

. 1997. Reconnaissances du travail - pour une approche ergologique. Paris: PUF.

SouZA-E-SiLva, M.C.P. de, D. FAÏTA 2002. Linguagem e Trabalho - construção de objetos de análise no Brasil e na França. São Paulo: Cortez. 